\title{
Erratum: Chiral Spin-Wave Velocities Induced by All-Garnet Interfacial Dzyaloshinskii-Moriya Interaction in Ultrathin Yttrium Iron Garnet Films [Phys. Rev. Lett. 124, 027203 (2020)]
}

\begin{abstract}
Hanchen Wang, Jilei Chen, Tao Liu, Jianyu Zhang, Korbinian Baumgaertl, Chenyang Guo, Yuehui Li, Chuanpu Liu, Ping Che, Sa Tu, Song Liu, Peng Gao, Xiufeng Han, Dapeng Yu, Mingzhong Wu, Dirk Grundler, and Haiming Yu॰
\end{abstract}

(Received 14 January 2022; published 27 January 2022)

DOI: 10.1103/PhysRevLett.128.049902

In this Letter, the sign of the magnetic field is found to be incorrect after the calibration of the magnetic field system, which does not alter the key observations of the Letter. All the signs of the applied magnetic field in the main text and supplemental materials should be reversed, which will influence the sign of the extracted interfacial Dzyaloshinskii-Moriya interaction (DMI) with its strength unchanged. During the calibration process, we used two independent methods to verify the applied field direction. First, we used the Hall sensor of the Gauss meter (Lake Shore 475) to measure the field direction. In the second method, we simply place a magnetic needle with north and south poles in the magnetic field to test its sign. Both methods verify that the sign used in the Letter should be reversed.

Thus, the DMI constant for 7-nm-thick yttrium iron garnet (YIG) film should be $-16 \mu \mathrm{J} / \mathrm{m}^{2}$ instead of $16 \mu \mathrm{J} / \mathrm{m}^{2}$. In this case, the sign of the DMI observed in this work is consistent with that of the recently reported DMI observed in 30-nm-thick YIG by nonlocal magnon spin transport [1].

[1] R. Schlitz, S. Vélez, A. Kamra, C. H. Lambert, M. Lammel, S. T. B. Goennenwein, and P. Gambardella, Control of Nonlocal Magnon Spin Transport via Magnon Drift Currents, Phys. Rev. Lett. 126, 257201 (2021). 